\title{
Heat and Mass transfer in MHD Nanofluid over a Stretching Surface along with Viscous Dissipation Effect
}

\author{
K. Govardhan \\ Department of Mathematics, \\ GITAM University, Hyderabad, India. \\ G. Narender \\ Department of Humanities and Sciences, \\ CVR College of Engineering, Hyderabad, India. \\ Corresponding author: gnriimc@gmail.com

\section{G. Sreedhar Sarma} \\ Department of Humanities and Sciences, \\ CVR College of Engineering, Hyderabad, India.
}

(Received December 24, 2018; Accepted August 29, 2019)

\begin{abstract}
A study of viscous dissipation effect of magnetohydrodynamic nanofluid flow passing over a stretched surface has been analyzed numerically. The formulated highly nonlinear equations for the above-mentioned flow are converted into first order ODEs. Utilizing the shooting technique along with the Adams-Bashforth Moulton Method is used to solve the BVP by using the computational software FORTRAN. The numerical results are computed by choosing different values of the involved physical parameters and compared with the earlier published results. The graphical numerical results of different physical quantities of interest are presented to analyze their dynamics under the varying physical quantities.
\end{abstract}

Keyword- Adams-Bashforth Moulton method, Thermophoresis, Brownian motion, Viscous dissipation, Nanofluids.

\section{Introduction}

The transfer of heat by the movement of fluids from one place to another is called convective heat transfer. Convective heat transfer is combination of heat diffusion and bulk fluid flow that are called conduction and advection. Large number of investigations on nanofluids (i.e. mixture of fluid and nanoparticles) show that can be improved thermal conductivity of base pure fluids. Nanofluid is a fluid containing nanometer-sized particles which are called as nanoparticles. These nanoparticles are made of metals, oxides, carbides etc.

There are countless industrial applications of heat and mass transfer. The applications of mass transfer are petrochemical refining, fractional distillation, where part of crude oil is separated. In short, every process of refining leverage mass transfer. Likewise, the heat transfer has a number of industrial applications e.g. in food industry meat and poultry processing, snack foods and in chemical industry for batch reactor, continuous processes and in asphalt and concrete industry for concrete heating and hot mix paving and in industrial laundry for at work ironers and steam generators. In many other industries, the process of heat and mass transfer over a stretching sheet is being used, like glass fiber production, aerodynamic extrusion of plastic sheets, glass blowing etc. In all these applications, the obtained products quality mainly depends upon the heat transfer rate at the stretching surfaces. Many authors like Bhatti and Rashidi (2017), Devi and Ganga 
International Journal of Mathematical, Engineering and Management Sciences

Vol. 5, No. 2, 343-352, 2020

https://doi.org/10.33889/IJMEMS.2020.5.2.028

(2009), Raju et al. (2016) discussed the heat and mass transfer and their applications. The applications of the transfer of heat and mass on MHD flow was canvassed by Mansour et al. (2000). Choi and Eastman (1995) studied the enhancing thermal conductivity of fluid with nanoparticles. The different theories of heat transfer in nanofluids are discussed by Buongiorno (2006). Kuznetsov and Nield (2010) studied the convective nanofluid in vertical plate, later they extended their work for porous medium. Khan and Pop (2011) explained the work on boundary layer flow of nanofluid through a stretching sheet. Heat transfer is the thermal energy movement from one system to another system at various temperatures. There are three mechanisms of transfer of heat: convection (through fluid movement), radiation (through electromagnetic waves), and conduction (through direct contact). It is a well-known fact that the phenomenon of heat transfer occurs between two bodies (or within the same body) due to the difference of temperature. The magnetic field or the magnetic surfaces play an important role in MHD. For example, along the direction of the magnetic field, the charged particles and the conducting fluid both can move easily but seldomly against it. Also, there are some functional variables on which the stability and confinement properties of fluids depend, and these variables are uniform in magnetic configuration. We will see how the fluid behaves in the presence of the magnetic field at different angles. The inclined magnetic field effects on fluid flow were explored by Seth et al. (2012), Singh et al. (2016), Dar (2017).

In different devices, the effect of viscous dissipation plays an important role in regular convection. The process in which the work has been done by fluid, which is also converted into heat is called viscous dissipation. It expresses the relationship between a flow's kinetic energy and enthalpy and is used to characterize the dissipation. Thermal boundary layer flow along with the effects of viscous dissipation and Ohmic heating over a heated plate were studied by Makinde and Mutuku (2014). Naramgari and Sulochana (2016) outlined the mass and heat transfer of the thermophoretic fluid flow past an exponentially stretched surface inserted in porous media in the presence of internal heat generation/absorption, infusion and viscous dissemination. Afify (2004) examined the MHD free convective heat and fluid flow passing over the stretched surface with chemical reaction. A numerical analysis of insecure MHD boundary layer flow of a nanofluid past a stretched surface in a porous media was carried out by Beg et al. (2014). Nadeem and Haq (2014) studied the magnetohydrodynamic boundary layer flow with the effect of thermal radiation over a stretching surface with the convective boundary conditions.

In this paper, some new effects which are not discussed yet in published work of Noghrehabadadi et al. (2012), has been discussed. The effects of inclined magnetic field, and activation energy on the two-dimensional hydromagnetic heat and mass transfer flow over a stretching sheet with viscous dissipation have been discussed.

\section{Mathematical Modeling}

A two-dimensional boundary layer flow of a study, viscous and incompressible nanofluid flow through a plate in a porous medium has been considered with focus on the heat and mass transfer. The fluid is assumed to flow above and along $x$-axis such that $y$-axis is perpendicular to the fluid motion. Two equal and opposite forces are brought into action by stretching the sheet to generate the flow. The sheet is stretched so that the velocity at each instant is proportional to the distance from its origin $(\mathrm{x}=0)$. The fluid is flowing under the influence of the magnetic field as displayed in Figure 1.

The flow is described by the equation of continuity, equation of momentum, energy equation and species equation as: 
International Journal of Mathematical, Engineering and Management Sciences

Vol. 5, No. 2, 343-352, 2020

https://doi.org/10.33889/IJMEMS.2020.5.2.028

\section{Continuity Equation}

Physical principal: Mass is conserved

$\frac{\partial \mathrm{u}}{\partial \mathrm{x}}+\frac{\partial \mathrm{v}}{\partial \mathrm{y}}=0$

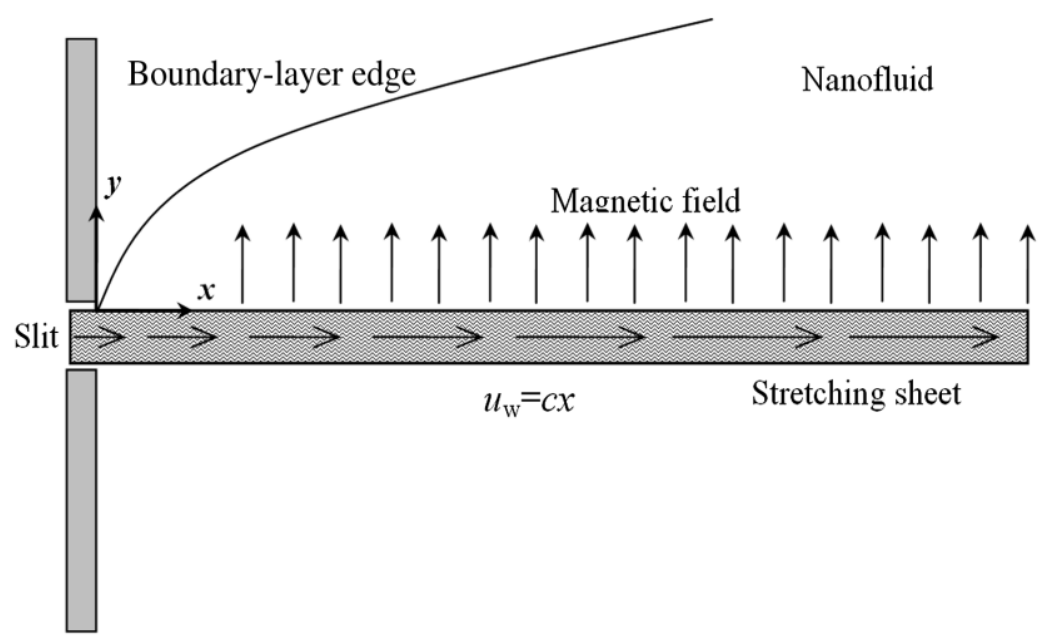

Figure 1. Geometry

\section{Momentum Equation}

Physical principle: $\mathrm{F}=\mathrm{ma}$

$u \frac{\partial \mathrm{u}}{\partial \mathrm{x}}+\mathrm{v} \frac{\partial \mathrm{u}}{\partial \mathrm{y}}=-\frac{1}{\rho_{f}} \frac{\partial p}{\partial y}+v\left(\frac{\partial^{2} u}{\partial x^{2}}+\frac{\partial^{2} u}{\partial y^{2}}\right)-\frac{\sigma B^{2}}{\rho_{f}} u$

In equation 2 . The components of velocity along $x$ and $y$ axis are respectively $u$ and $v, p$ the fluid pressure, $\rho_{f}$ the nanofluid density, $\sigma$ is conductivity of materials, $v$ the kinematic viscosity of the base fluid and $B$ is the magnetic induction.

\section{Energy Equation}

Physical principle: Energy is conserved. During, using the boundary layer approximations, the boundary layer equation of energy for fluid temperature $T$ is

$u \frac{\partial \mathrm{T}}{\partial \mathrm{x}}+\mathrm{v} \frac{\partial \mathrm{T}}{\partial \mathrm{y}}=\alpha \frac{\partial^{2} T}{\partial y^{2}}+\tau\left[D_{B}\left(\frac{\partial \mathrm{T}}{\partial \mathrm{y}} \frac{\partial \mathrm{C}}{\partial \mathrm{y}}\right)+\left(\frac{D_{T}}{T_{\infty}}\right)\left(\frac{\partial \mathrm{T}}{\partial \mathrm{y}}\right)^{2}\right]+\frac{\mu}{\rho_{p}}\left[\left(\frac{\partial u}{\partial y}\right)^{2}\right]$

In Equation (3), the third term on the right-hand side is associated with viscous dissipation, while the second term on the right-hand side captures the convective transport due to Brownian motion and thermophoresis.

\section{Mass Transfer Equation}

$u \frac{\partial \mathrm{C}}{\partial \mathrm{x}}+\mathrm{v} \frac{\partial \mathrm{C}}{\partial \mathrm{y}}=D_{B} \frac{\partial^{2} C}{\partial y^{2}}+\left(\frac{D_{T}}{T_{\infty}}\right) \frac{\partial^{2} T}{\partial y^{2}}$ 
International Journal of Mathematical, Engineering and Management Sciences

Vol. 5, No. 2, 343-352, 2020

https://doi.org/10.33889/IJMEMS.2020.5.2.028

The associated boundary conditions for the above system of equations are,

$$
\left.\begin{array}{c}
u=U_{w}(x), v=0, T=T_{w}, C=C_{w}, \text { at } y=0 \\
u=v=0, T=T_{\infty}, C=C_{\infty}, \text { as } y \rightarrow \infty
\end{array}\right\}
$$

In above equations, $T$ is the temperature of the nanofluids and the ambient temperature is $T_{\infty}$ respectively. $C$ is nanoparticles concentration, $C_{\infty}$ shows the free stream concentration. Brownian diffusion coefficient $D_{B}, D_{T}$ denotes the thermophoretic diffusion coefficient

\section{Non-dimensional Form of the Governing Equations}

Suitable similarity transformation has been used for the conversion of the mathematical model (1), (2), (3) and (4) along with the boundary conditions (5) from dimensional form to non-dimensional form. The following similarity transformation as defined in (2012) has been used.

$\eta=y \sqrt{\frac{c}{v}}, \quad \psi=\sqrt{c v} x f(\eta), \quad \theta(\eta)=\frac{T-T_{\infty}}{T_{f}-T_{\infty}}, \quad \beta(\eta)=\frac{C-C_{\infty}}{C_{w}-C_{\infty}}$.

In above, $\psi(x, y)$ denotes stream function obeying

$u=\frac{\partial \psi}{\partial y}, v=-\frac{\partial \psi}{\partial x}$

By introducing the similarity transformation defined as above, equations (2) - (4) becomes:

$$
\begin{aligned}
& f^{\prime \prime \prime}+f f^{\prime \prime}-f^{\prime 2}-M f^{\prime}=0 \\
& \theta^{\prime \prime}+\operatorname{Pr}\left(f \theta^{\prime}+N b \theta^{\prime} \beta^{\prime}+N t \theta^{2}+E c f^{\prime \prime 2}\right) \\
& \beta^{\prime \prime}+L e f \beta^{\prime}+\frac{N t}{N b} \theta^{\prime \prime}=0
\end{aligned}
$$

The associated boundary conditions $(5)$ get the form:

$$
\left.\begin{array}{c}
\eta=0, f(\eta)=0, f^{\prime}(\eta)=1, \theta(\eta)=1, \beta(\eta)=1 \text { at } \eta=0, \\
\eta \rightarrow \infty, f^{\prime}(\infty) \rightarrow 0, \theta(\infty) \rightarrow 0, \beta(\infty) \rightarrow 0 .
\end{array}\right\}
$$

Different parameters used in the above equations have the following formulations:

$$
\begin{aligned}
\operatorname{Pr} & =\frac{v}{\alpha}, \quad E c=\frac{u_{w}^{2}}{\left[\left(C_{p}\right)_{f}\left(T_{w}-T_{\infty}\right)\right]}, \quad L e=\frac{v}{D_{B}}, \quad N b=\frac{(\rho c)_{p} D_{B}\left(C_{w}-C_{\infty}\right)}{(\rho c)_{f} v}, \quad N t=\frac{(\rho c)_{p} D_{T}\left(T_{w}-C T_{\infty}\right)}{(\rho c)_{f} v T_{\infty}}, \\
M & =\frac{\sigma B^{2}}{(\rho c)_{f}}
\end{aligned}
$$

where $\operatorname{Pr}$ denotes the Prandtl number, $E c$ is the Eckert number, Le is the Lewis number, $N b$ the Brownian motion parameter, $N t$ the thermophoresis parameter, and $M$ is the magnetic parameter. The skin friction coefficient $C_{f}$, Nusselt number $N u_{x}$ and Sherwood number $S h_{x}$ are defined as

$$
C_{f}=\frac{\tau_{w}}{\rho u_{w}^{2}}, N u_{x}=\frac{x q_{w}}{k\left(\left(T_{w}-T_{\infty}\right)\right.}, S h_{x}=\frac{x h_{m}}{D_{B}\left(\left(\varphi_{w}-\varphi_{\infty}\right)\right.}
$$

Here, the heat flux $q_{w}$, the skin - friction on flat plate $\tau_{w}$, and the mass transfer rate $h_{w}$, are given by

$$
q_{w}=-k\left(\frac{\partial T}{\partial y}\right)_{y=0}, \tau_{w}=\left(\mu_{\mathrm{B}}+\frac{p_{y}}{\sqrt{2 \pi_{c}}}\right)\left(\frac{\partial u}{\partial y}\right)_{y=0}, h_{m}=-D_{B}\left(\frac{\partial \varphi}{\partial y}\right)_{y=0}
$$


International Journal of Mathematical, Engineering and Management Sciences

Vol. 5, No. 2, 343-352, 2020

https://doi.org/10.33889/IJMEMS.2020.5.2.028

By using the above equations, we have

$C_{f} \sqrt{R_{x}}=-f^{\prime \prime}(0), \frac{N u_{x}}{\sqrt{R_{x}}}=-\theta^{\prime}(0), \frac{S h_{x}}{\sqrt{R_{x}}}=-\beta^{\prime}(0)$

where $R_{x}=a x^{2}$ is the local Reynolds number.

\section{Numerical Treatment}

The set of non-linear coupled differential equations (8) - (10) with the conditions (11) is solved numerically in the following manner. Initially, it is noticed that heurist infinity for the independent variable chosen as $\eta_{\max }$. The comment on the choice on the $\eta_{\max }$, for solving is presented at the end of the section.

Equation (8) is solved with $f^{\prime \prime}(0)=r$, assumed number using the initial conditions $f(0)=0, f^{\prime}(0)=1, f^{\prime \prime}(0)=r$

$r$ is iteratively found using Newton's method using $G^{\prime}\left(\eta_{\max }\right)=\frac{\partial f^{\prime}(\max )}{\partial \alpha}$ which is obtain by solving,

$G^{\prime \prime \prime}=2 f^{\prime} G^{\prime}-f G^{\prime \prime}-f^{\prime \prime} G^{\prime}+M f^{\prime}$

with $G(0)=0, G^{\prime}(0)=0$ and $G^{\prime \prime}(0)=1$

After finding $f(\eta)$ we solved the equations (9) \& (10) with the initial conditions.

$$
\left.\begin{array}{rr}
\mathrm{y}_{1}^{\prime}=\mathrm{y}_{2}, & \mathrm{y}_{1}(0)=1 \\
\mathrm{y}_{2}^{\prime}=-\operatorname{Pr}\left(f(\eta) \mathrm{y}_{2}+\mathrm{Nby}_{2} \mathrm{y}_{4}+\mathrm{Nt}\left(y_{2}\right)^{2}+\mathrm{Ec}\left(f^{\prime \prime}\right)^{2}\right), & \mathrm{y}_{2}(0)=\mathrm{p}_{1} \\
\mathrm{y}_{3}^{\prime}=\mathrm{y}_{4}, & \mathrm{y}_{3}(0)=1 \\
\mathrm{y}_{4}^{\prime}=-\operatorname{Le} f(\eta) \mathrm{y}_{4}-\frac{\mathrm{Nb}}{\mathrm{Nt}} \mathrm{y}_{2}^{\prime}, & \mathrm{y}_{4}(0)=\mathrm{p}_{2}
\end{array}\right\}
$$

Here $p_{1}=\theta^{\prime}(0)$ and $p_{2}(0)=\beta^{\prime}(0)$.

$p_{1}, p_{2}$ are to be found satisfying end conditions $y_{1} \rightarrow 0, y_{3} \rightarrow 0$ as $\eta \rightarrow \infty$. Adams Moultan fourth order method (with the corresponding predictor) is used to solve the initial value problem. Assumed values of $p_{1}$ and $p_{2}$ are corrected using Newton method.

Derivatives of $\theta\left(\infty, p_{1}, p_{2}\right)$ and $\beta\left(\infty, p_{1}, p_{2}\right)$ with respect to any parameter $p\left(p_{1}\right.$ or $\left.p_{2}\right)$ are found by solving the equation which are obtained by differentiating system (16).

$Y_{i}=\frac{\partial y_{i}}{\partial p}$ for all $i=1,2,3,4$

These equations are 
International Journal of Mathematical, Engineering and Management Sciences

Vol. 5, No. 2, 343-352, 2020

https://doi.org/10.33889/IJMEMS.2020.5.2.028

$$
\begin{aligned}
& Y_{1}^{\prime}=Y(2) \\
& Y_{2}^{\prime}=-\operatorname{Pr}[f(\eta) Y(2)+N b(y(2) Y(4)-Y(2) y(4))+2 N t y(2) Y(2)] \\
& Y_{3}^{\prime}=Y(4) \\
& Y_{4}^{\prime}=-\operatorname{Lef}(\eta) y(4)-\frac{N t}{N b} Y_{2}^{\prime}
\end{aligned}
$$

This system is solved with three different sets of initial conditions $y_{i}(0)=0$ for all $i=1,2,3,4$. Newton's method is

$\left(\begin{array}{l}p_{1} \\ p_{2}\end{array}\right)^{\text {New }}=\left(\begin{array}{l}p_{1} \\ p_{2}\end{array}\right)^{\text {old }}-\left[\begin{array}{ll}\frac{\partial y_{1}}{\partial p_{1}} & \frac{\partial y_{1}}{\partial p_{2}} \\ \frac{\partial y_{3}}{\partial p_{1}} & \frac{\partial y_{3}}{\partial p_{2}}\end{array}\right]_{\eta=\infty}^{-1}\left(\begin{array}{l}y_{1} \\ y_{3}\end{array}\right)$

It may be noticed that the choice of initial guess of $p_{1}, p_{2}$ is very crucial. Once we obtain solution for a set of physical parameters, a single parameter changed slightly to achieve convergence of newton's method. The convergence criteria is chosen to be successive value which agrees up to 3 significant digits. The choice of $\eta_{\max }=4$ was more than enough for end condition.

\section{Results and Discussion}

In order to validate the code adopted for the numerical solution of equations governing the natural convective flow, the comparison of current results with some of the earlier published work on free

\begin{tabular}{|c|c|c|c|c|c|c|c|c|c|}
\hline \multirow[b]{2}{*}{$N t$} & \multirow[b]{2}{*}{$N b$} & \multicolumn{4}{|c|}{$-\boldsymbol{\theta}^{\prime}(\mathbf{0})$} & \multicolumn{4}{|c|}{$-\boldsymbol{\beta}^{\prime}(\mathbf{0})$} \\
\hline & & $\begin{array}{c}\text { Khan } \\
\text { and } \\
\text { Pop } \\
\text { (2011) }\end{array}$ & $\begin{array}{c}\text { Beg et al. } \\
\text { (2014) }\end{array}$ & $\begin{array}{l}\text { Noghrehabadadi } \\
\qquad(2012)\end{array}$ & Present Result & $\begin{array}{c}\text { Khan } \\
\text { and } \\
\text { Pop } \\
\text { (2011) }\end{array}$ & $\begin{array}{c}\text { Beg et al. } \\
\text { (2014) }\end{array}$ & $\begin{array}{l}\text { Noghrehabadadi } \\
\qquad \text { (2012) }\end{array}$ & $\begin{array}{l}\text { Present } \\
\text { Result }\end{array}$ \\
\hline 0.1 & 0.1 & 0.9524 & 0.9523768 & 0.9523767 & 0.952376800 & 2.1294 & 2.1293938 & 2.1293938 & 2.129389000 \\
\hline & 0.2 & 0.6932 & 0.6931743 & 0.6931743 & 0.693174600 & 2.2740 & 2.2740215 & 2.2740215 & 2.274012000 \\
\hline & 0.3 & 0.5201 & 0.5200790 & 0.5200790 & 0.520079700 & 2.5286 & 2.5286382 & 2.5286381 & 2.528624000 \\
\hline & 0.4 & 0.4026 & 0.4025808 & 0.4025810 & 0.402581500 & 2.7952 & 2.7951701 & 2.7951703 & 2.795155000 \\
\hline & 0.5 & 0.3211 & 0.3210543 & 0.3210544 & 0.321055100 & 3.0351 & 3.0351425 & 3.0351425 & 3.035121000 \\
\hline 0.2 & & 0.5056 & 0.5055814 & 0.5055814 & 0.505581300 & 2.3819 & 2.3818706 & 2.3818707 & 2.381866000 \\
\hline 0.3 & & 0.2522 & 0.2521560 & 0.2521560 & 0.252155500 & 2.4100 & 2.4100188 & 2.4100188 & 2.410016000 \\
\hline 0.4 & & 0.1194 & 0.1194059 & 0.1194059 & 0.119405400 & 2.3997 & 2.3996502 & 2.3996502 & 2.399647000 \\
\hline 0.5 & & 0.0543 & 0.0542534 & 0.0542534 & 0.054252970 & 2.3836 & 2.3835712 & 2.3835712 & 2.383568000 \\
\hline
\end{tabular}
convection are displayed in Table 1. Excellent agreement of current results with those previously published results encourage us to use the present code.

Table 1. Comparison of the results of $-\theta^{\prime}(0) \&-\beta^{\prime}(0)$ when $\operatorname{Pr}=L e=10, E c=0$, and $M=0$ 
International Journal of Mathematical, Engineering and Management Sciences

Vol. 5, No. 2, 343-352, 2020

https://doi.org/10.33889/IJMEMS.2020.5.2.028

Figures 2, 3, 4 and 5 show the effect of magnetic parameter on temperature distribution and concentration distribution respectively. It can be seen from the figures that boundary layer thickness increases with the increase in magnetic parameter. It is due to the Lorentz force which is created by applying magnetic field to the conducting fluid. Lorentz force has the tendency to reduce the speed of flow which supports our results. By applying magnetic field on the fluid, the resistance on the fluid particles increases which results in the increase in temperature.

Figures 2 and 3 represent the effect of Brownian motion, parameter and thermophoresis parameter for a variation of the magnetic parameter of $M$ on temperature and concentration profile. An increase in Brownian motion parameter increases the temperature. Similarly increase in thermophoresis parameter increases the concentration distribution. Thermophoresis is a component that drives small materials away from hot layer to the cooler end. Furthermore, the magnetic field strongly influences the areas that are far from the wall when $N b$ and $N t$ are comparatively high. Figure 4 and 5 displays the influence of Eckert number $E c$ on the energy and mass transfer profiles.

It is observed that energy profile and mass transfer profiles increase when the Eckert number is increased. Due to friction, the heat energy is kept in owing to accelerating values of Eckert number, which results in the enhancement of the temperature and concentration profile.

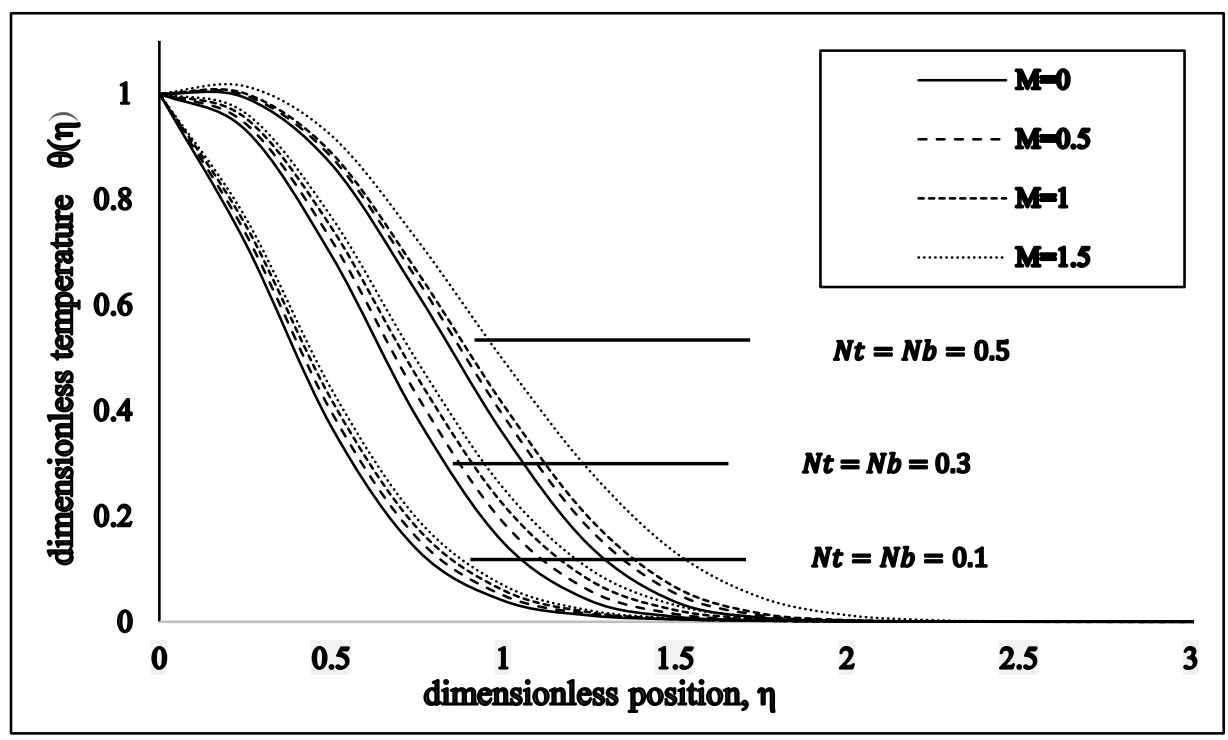

Figure 2. Impact of $M, N b$, and $N t$ when $P r=L e=10$, and $E c=0.1$ on Temperature profile. 
International Journal of Mathematical, Engineering and Management Sciences

Vol. 5, No. 2, 343-352, 2020

https://doi.org/10.33889/IJMEMS.2020.5.2.028

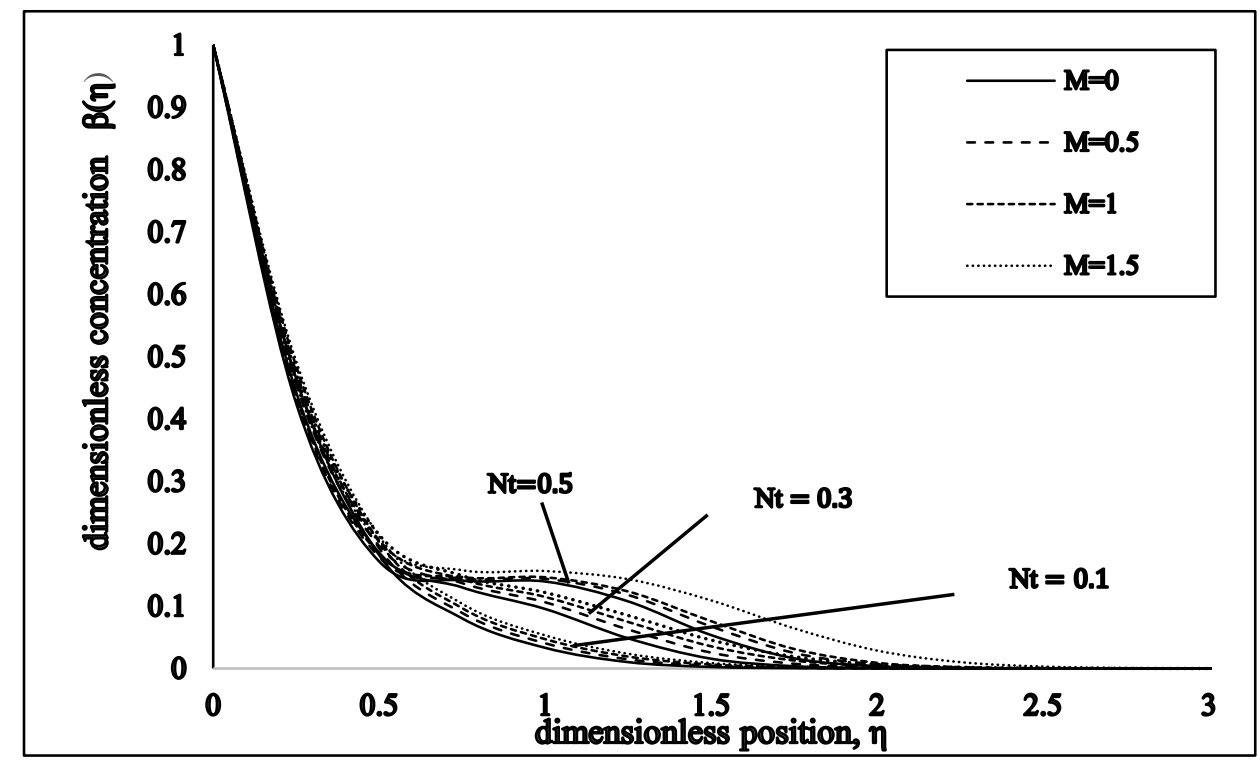

Figure 3. Impact of $M$, and $N t$, when $\operatorname{Pr}=L e=10, N b=0.5$ and $E c=0.1$ on concentration profile.

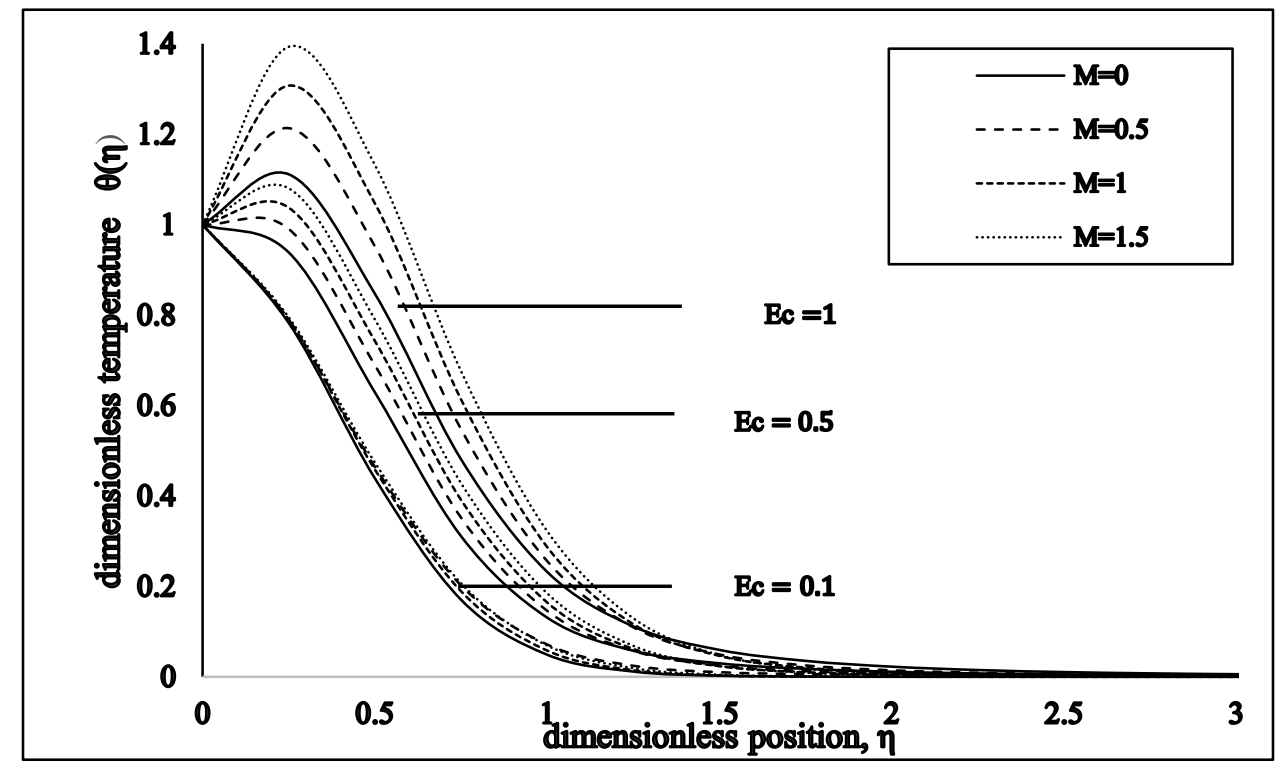

Figure 4. Impact of $M$ and $E c$ when $\operatorname{Pr}=L e=10, N b=0.2$ and $N t=0.1$ on Temperature profile. 
International Journal of Mathematical, Engineering and Management Sciences

Vol. 5, No. 2, 343-352, 2020

https://doi.org/10.33889/IJMEMS.2020.5.2.028

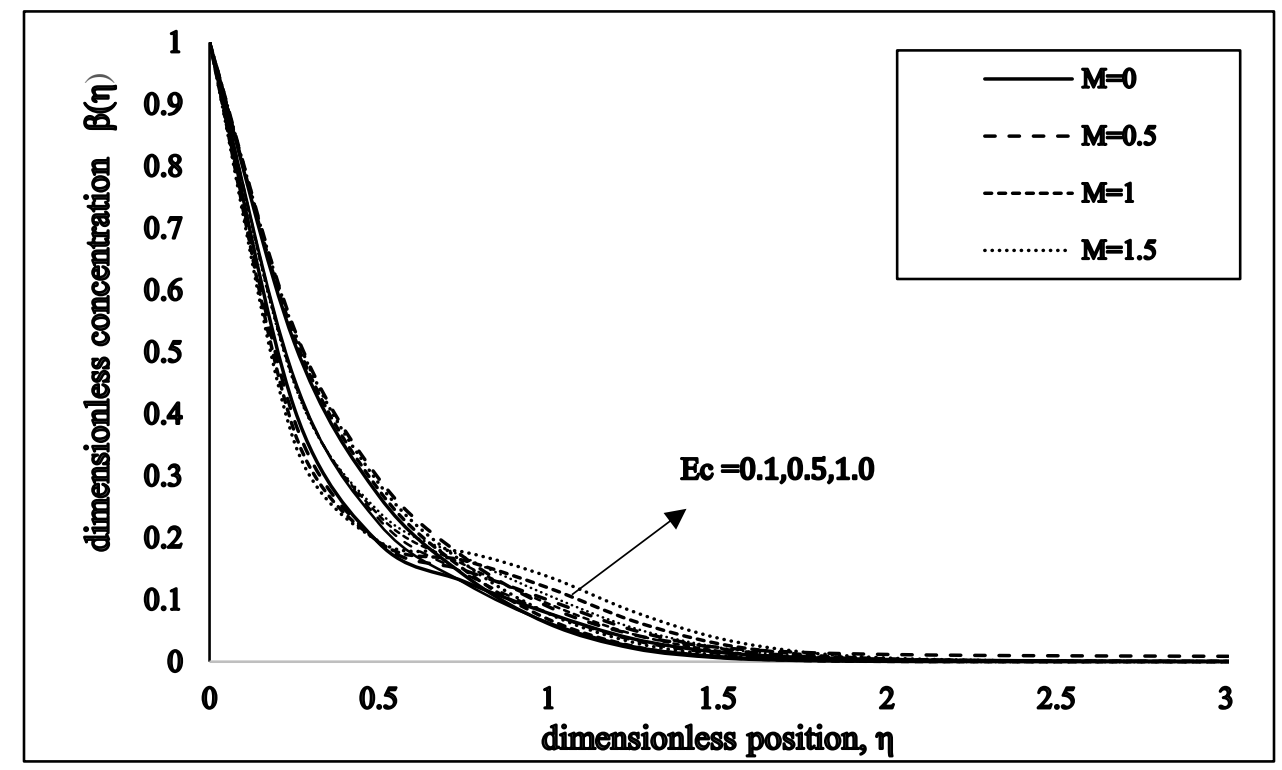

Figure 5. Impact of of $M$ when $\operatorname{Pr}=L e=10, N b=0.2, E c=0.1$ and $N t=0.1$ on concentration profile.

\section{Conclusion}

The Nusselt number decreases for increasing values of the thermophoresis parameter $N t$ where as Sherwood number increases for increasing values of Brownian motion parameter. It is reported that enhancing values of the MHD parameter $M, N t$ and $N b$ thermal boundary-layer thickness also enhanced. It is observed that temperature profile and concentration profile show an increment for growing values of dimensionless parameter $N t$. Physically, heated particles comes away from high temperature as compared to low-temperature so the temperature of fluid increases. The increase of Eckert number shows that temperature profile is expanding, and concentration profile observed for increasing values of increases $E c$ and $M$. The physical reason behind it is that an increment in the dissipation enhances the thermal conductivity of the fluid which causes an enhancement in the thermal boundary layer. The concentration profile is enhanced for the gradually mounting values of thermophoresis parameter.

\section{Conflict of Interest}

The author confirms that this article contents have no conflict of interest.

\section{Acknowledgement}

Help by Prof. S. R. Koneru, Retd. Professor, Department of Mathematics, IIT Mumbai in the preparation of this paper is gratefully acknowledged.

\section{References}

Afify, A.A. (2004). MHD free convective flow and mass transfer over a stretching sheet with chemical reaction. Heat and Mass Transfer, 40(6-7), 495-500. 
International Journal of Mathematical, Engineering and Management Sciences

Vol. 5, No. 2, 343-352, 2020

https://doi.org/10.33889/IJMEMS.2020.5.2.028

Beg, O.A., Khan, M.S., Karim, I., Alam, M.M., \& Ferdows, M. (2014). Explicit numerical study of unsteady hydromagnetic mixed convective nanofluid flow from an exponentially stretching sheet in porous media. Applied Nanoscience, 4(8), 943-957.

Bhatti, M.M., \& Rashidi, M.M. (2017). Study of heat and mass transfer with joule heating on magnetohydrodynamic (MHD) peristaltic blood flow under the influence of hall effect. Propulsion and Power Research, 6(3), 177-185.

Buongiorno, J. (2006). Convective transport in nanofluids. Journal of Heat Transfer, 128 (3), 240-250.

Choi, S.U.S., \& Eastman, J.A. (1995). Enhancing thermal conductivity of fluids with nanoparticles. (No. ANL/MSD/CP-84938; CONF-951135-29). Argonne National Lab., IL (United States).

Dar, A.A. (2017). Effect of an inclined magnetic field on the flow of nanofluids in a tapered asymmetric porous channel with heat source/sink and chemical reaction. Applied Science and Innovative Research, 1(2), 104-117.

Devi, S.A., \& Ganga, B. (2009). Effects of viscous and Joules dissipation on MHD flow, heat and mass transfer past a stretching porous surface embedded in a porous medium. Nonlinear Analysis. Modeling and Control, 14(3), 303-314.

Khan, W.A., \& Pop, I. (2011). Flow and heat transfer over a continuously moving at plate in a porous medium. Journal of Heat Transfer, 133(5), 054501(1-5).

Kuznetsov, A.V., \& Nield, D.A. (2010). Natural convective boundary-layer flow of a nanofluid past a vertical plate. International Journal of Thermal Sciences, 49(2), 243-247.

Makinde, O.D., \& Mutuku, W.N. (2014). Hydromagnetic thermal boundary layer of nanofluids over a convectively heated at plate with viscous dissipation and Ohmic heating. Universitatea Politecnica Bucuresti Scientific Bulletin Series A, 76(2), 181-192.

Mansour, M.A., El-Hakiem, M.A., \& El Kabeir, S.M. (2000). Heat and mass transfer in magnetohydrodynamic flow of micropolar fluid on a circular cylinder with uniform heat and mass flux. Journal of Magnetism and Magnetic Materials, 220, 259-270.

Nadeem, S., \& Haq, R.U. (2014). Effect of thermal radiation for magnetohydrodynamic boundary layer flow of a nanofluid past a stretching sheet with convective boundary conditions. Journal of Computational and Theoretical Nanoscience, 11(1), 32-40.

Naramgari, S., \& Sulochana, C. (2016). Dual solutions of radiative MHD nanofluid flow over an exponentially stretching sheet with heat generation/absorption. Applied Nanoscience, 6(1), 131-139.

Noghrehabadadi, A., Ghalambaz, M., \& Ghanbarzadeh, A. (2012). Heat transfer of magnetohydrodynamic viscous nanofluids over an isothermal stretching sheet. Journal of Thermophysics and Heat Transfer, 26(4), 686-689.

Raju, C.S.K., Sandeep, N., Sugunamma, V., Babu, M.J., \& Reddy, J.R. (2016). Heat and mass transfer in magnetohydrodynamic casson fluid over an exponentially permeable stretching surface. Engineering Science and Technology, an International Journal, 19(1), 45-52.

Seth, G.S., Nandkeolyar, R., \& Ansari, M.S. (2012). Effects of hall current and rotation on unsteady MHD couette flow in the presence of an inclined magnetic field. Journal of Applied Fluid Mechanics, 5(2), $67-74$.

Singh, N.K., Kumar, V., \& Sharma, G.K. (2016). The effect of inclined magnetic field on unsteady flow past on moving vertical plate with variable temperature. International Journal of Latest Technology in Engineering, Management \& Applied Science, 5(2), 2278-2540.

Original content of this work is copyright $(\mathcal{C}$ International Journal of Mathematical, Engineering and Management Sciences. Uses under the Creative Commons Attribution 4.0 International (CC BY 4.0) license at https://creativecommons.org/licenses/by/4.0/ 